\title{
Late-onset Quetiapine-related Tardive Dyskinesia Side Effects in a Patient with Psychotic Depression
}

\author{
Yi-Cheng Hou', Chien-Han Lai ${ }^{2}$ \\ 'Department of Nutrition, Taipei Tzu Chi Hospital, Buddhist Tzu Chi Medical Foundation, New Taipei City, ${ }^{2}$ Department of Psychiatry, Cheng \\ Hsin General Hospital, Taipei City, Taiwan
}

\begin{abstract}
The atypical antipsychotics were believed to induce less extrapyramidal syndrome, including tardive dyskinesia (TD). Since the introduction of the quetiapine, it is also reported with less TD side effects. It even can relieve the symptoms of severe TD and reduce the risk of TD. The quetiapine's low affinity and fast dissociation from postsynaptic dopamine D2 receptors should give the least risk of producing the symptoms of TD. The quetiapine even can reduce the TD side effects related to clozapine, which has the lowest risk for TD. However, since the first case report of TD side effects related to quetiapine published on 1999 , the safety of quetiapine in TD aspect has been questioned. Therefore, we want to share this case report, which was written to describe the severe late-onset TD side effects after long-term use of quetiapine in a patient with psychotic depression. The patient had no significant findings after concurrent comprehensive neurological examinations, magnetic resonance imaging of brain and electroencephalogram since the onset of TD.
\end{abstract}

KEY WORDS: Tardive dyskinesia, Quetiapine, Psychotic depression.

\section{INTRODUCTION}

Atypical antipsychotics are believed to reduce the risk of extrapyramidal syndrome, including tardive dyskinesia (TD). Quetiapine is reported to be less likely to induce TD side effects and, in fact, has been shown to relieve symptoms of severe $\mathrm{TD}^{1)}$ and reduce TD risk. ${ }^{2)}$ Quetiapine's low affinity and fast dissociation from postsynaptic dopamine D2 receptors should contribute to low TD symptom production. ${ }^{3)}$ Additionally, quetiapine reduces TD side effects from clozapine, a drug already associated with very low TD risk. ${ }^{4)}$ However, since the first case report of quetiapine-related TD side effects was published in 1999, ${ }^{5)}$ quetiapine's safety has been questioned. This case report describes severe, late-onset TD side effects after longterm quetiapine use in a patient with psychotic depression. Since TD onset, the patient has had no significant findings based on comprehensive neurological examinations, brain magnetic resonance imaging, and electroencephalogram.

\footnotetext{
Received: January 24, 2014 / Revised: March 6, 2014

Accepted: March 19, 2014

Address for correspondence: Chien-Han Lai, MD

Department of Psychiatry, Cheng Hsin General Hospital, No.45,

Cheng Hsin St., Pai-Tou Destrict, Taipei City, Taiwan

Tel: +886-2-28264400 ext 3502, Fax: +886-2-28264570

E-mail: stephenlai99@gmail.com
}

\section{CASE}

The patient was a female homemaker who has suffered with psychotic depression for 3 years. Her symptoms included depressed mood, lack of interest, lack of energy, suicidal ideation, insomnia, feelings of worthlessness, auditory hallucinations (voices commenting around her ears and commanding her to die), and delusions of poverty. Diagnosis was based on criteria of the Diagnostic and Statistical Manual of Mental Disorders IV text revision (DSM-IV-TR) and the Structured Clinical Interview for DSM-IV-TR. Symptom severity was as follows: Brief Psychiatric Rating Scale-18 items (BPRS-18) score=31, especially for hallucinatory behaviors (5 points), and Hamilton Rating Scales for Depression (HRSD) score $=27$. Quetiapine and duloxetine were delivered using gradual titration to relieve symptoms, and the final stable medication doses were quetiapine $600 \mathrm{mg}$ /day and duloxetine $90 \mathrm{mg}$ /day across 2.5 years. The patient's depressive and psychotic symptoms responded to psychotropic medication and reached partial remission status (BPRS score=9; HRSD score=12). She did not have extrapyramidal symptoms during the first two years after initiating the above medications. However, she began to experience severe perioral tremors with involuntary, repetitive, 
and irregular movements of tongue starting in the third year of quetiapine treatment. The protruding tongue with irregular movements was a significant detriment to her quality of life, as irregular movements occurred all day and were not relieved by anticholinergic medications. Quetiapine-related TD was suspected, and the quetiapine dose was tapered to $150 \mathrm{mg} /$ day. However, after 3 months, TD severity remained the same and seemed unresponsive to interventions, including switching to other atypical antipsychotics such as aripiprazole or ziprasidone. No significant exacerbations of psychotic or mood symptoms were observed after TD onset and antipsychotic switch. The patient also received concurrent comprehensive neurological examinations, brain magnetic resonance imaging, and electroencephalograms. No significant alterations in brain anatomy or function were observed, and according to the neurological report, no organic etiology could be found to explain the presence of TD.

\section{DISCUSSION}

In this case, we observed unique, late-onset, and irreversible TD side effects after long-term quetiapine use. Late-onset TD after 3 years might suggest independent factors. However, comprehensive neurological examinations and imaging studies could not pinpoint organic evidence to suggest that this patient's TD was independent of quetiapine use. Late-onset TD side effects in this patient appear irreversible even after decreasing quetiapine dose and switching antipsychotics. However, case reports of late-onset TD have not been included in previous reports. Rather, these reports mention the long-term safety of quetiapine in adolescent ${ }^{6)}$ and adult patients. ${ }^{3)}$ Sacchetti and Valsecchi ${ }^{7)}$ showed that quetiapine has the lowest risk for TD side effects when compared with olanzapine and clozapine in their long-term trial (154 weeks). Quetiapine-related TD was noted and described in an early case report about 15 years ago. ${ }^{5)}$ However, some reports also mention contrary findings of quetiapine's ability to relieve TD. ${ }^{1,2,8-13)}$ Emsley et al. ${ }^{14)}$ found that quetiapine could effectively reduce TD severity in patients with established $\mathrm{TD}$, but the mechanism of this action is unclear. Quetiapine is the atypical antipsychotic most similar to clozapine (without its hematologic side effects) based on receptor and pharmacologic profile, which may explain its treatment effects for TD. ${ }^{11)}$ Quetiapine is believed to express lower affinity for D2 receptors in striatal and extrastriatal regions of brain, ${ }^{15)}$ which could explain the mechanisms by which it can treat or relieve TD side effects. The presence of mood disorders may be a possible reason for quetiapine-related TD in this patient. Sharma ${ }^{16)}$ reported that patients with mood disorders were more prone to treatment-emergent TD with quetiapine. Recently, reports reveal quetiapine's TD side effects and suggest precautionary attention to TD risk characteristics. ${ }^{17)} \mathrm{A}$ recent dopamine receptor imaging study showed that quetiapine occupies dopamine D2 receptors extensively in striatal regions, which likely contributes to TD side effects. ${ }^{18)}$ Furthermore, low-dose quetiapine also seems to induce early-onset TD in the neuroleptic-naïve patient. ${ }^{19)}$ Recent reports provide evidence of quetiapine's TD risk as well as possible mechanisms. Previously, it was suggested that quetiapine-related TD could be relieved by tapering the dose or switching to another atypical antipsychotic such as aripiprazole. ${ }^{15)}$ However, in this case study, TD was not abated with quetiapine dose tapering or with a switch to other atypical antipsychotics. Therefore, use of quetiapine should be approached with caution, with close attention paid to TD risk factors.

\section{REFERENCES}

1. Vesely C, Küfferle B, Brücke T, Kasper S. Remission of severe tardive dyskinesia in a schizophrenic patient treated with the atypical antipsychotic substance quetiapine. Int Clin Psychopharmacol 2000;15:57-60.

2. Farah A. Reduction of tardive dyskinesia with quetiapine. Schizophr Res 2001;47:309-310.

3. Adityanjee, Schulz SC. Clinical use of quetiapine in disease states other than schizophrenia. J Clin Psychiatry 2002;63 (Suppl 13):32-38.

4. Alptekin K, Kivircik BB. Quetiapine-induced improvement of tardive dyskinesia in three patients with schizophrenia. Int Clin Psychopharmacol 2002;17:263-264.

5. Ghelber D, Belmaker RH. Tardive dyskinesia with quetiapine. Am J Psychiatry 1999;156:796-797.

6. McConville B, Carrero L, Sweitzer D, Potter L, Chaney R, Foster $\mathrm{K}$, et al. Long-term safety, tolerability, and clinical efficacy of quetiapine in adolescents: an open-label extension trial. J Child Adolesc Psychopharmacol 2003;13:75-82.

7. Sacchetti E, Valsecchi P. Quetiapine, clozapine, and olanzapine in the treatment of tardive dyskinesia induced by first-generation antipsychotics: a 124-week case report. Int Clin Psychopharmacol 2003;18:357-359.

8. Nelson MW, Reynolds RR, Kelly DL, Conley RR. Adjunctive quetiapine decreases symptoms of tardive dyskinesia in a patient taking risperidone. Clin Neuropharmacol 2003;26:297-298.

9. Sasaki Y, Kusumi I, Koyama T. A case of tardive dystonia successfully managed with quetiapine. J Clin Psychiatry 2004;65:583-584.

10. Bouckaert F, Herman G, Peuskens J. Rapid remission of severe tardive dyskinesia and tardive dystonia with quetiapine. Int J Geriatr Psychiatry 2005;20:287-288.

11. Gourzis P, Polychronopoulos P, Papapetropoulos S, Assimakopoulos K, Argyriou AA, Beratis S. Quetiapine in the treatment of focal tardive dystonia induced by other atypical 
antipsychotics: a report of 2 cases. Clin Neuropharmacol 2005;28:195-196.

12. Cortese L, Caligiuri MP, Williams R, Schieldrop P, Manchanda R, Malla A, et al. Reduction in neuroleptic-induced movement disorders after a switch to quetiapine in patients with schizophrenia. J Clin Psychopharmacol 2008;28:69-73.

13. Ono S, Suzuki Y, Shindo M, Endo T, Fukui N, Sugai T, et al. Improvement of tardive dyskinesia and dystonia associated with aripiprazole following a switch to quetiapine: case report and review of the literature. J Clin Pharm Ther 2012;37:370-372.

14. Emsley R, Turner HJ, Schronen J, Botha K, Smit R, Oosthuizen PP. A single-blind, randomized trial comparing quetiapine and haloperidol in the treatment of tardive dyskinesia. J Clin Psychiatry 2004;65:696-701.

15. Rizos E, Douzenis A, Gournellis R, Christodoulou C,
Lykouras LP. Tardive dyskinesia in a patient treated with quetiapine. World J Biol Psychiatry 2009;10:54-57.

16. Sharma V. Treatment-emergent tardive dyskinesia with quetiapine in mood disorders. J Clin Psychopharmacol 2003;23:415-417.

17. Traynor K. FDA advisers wary of expanding quetiapine use: clinicians air concerns about metabolic effects, tardive dyskinesia. Am J Health Syst Pharm 2009;66:880, 882.

18. Chatziioannou SN, Rizos EN, Kallergi M, Douzenis A, Apostolopoulos A, Bacalis S, et al. Tardive dyskinesia related to quetiapine and confirmed by a DAT scan. J Clin Psychopharmacol 2011;31:535-538.

19. Walsh RA, Lang AE. Early-onset tardive dyskinesia in a neuroleptic-naive patient exposed to low-dose quetiapine. Mov Disord 2011;26:2297-2298. 